\title{
TRADICIÓN Y CONTINUIDAD COMO INNOVACIÓN EDUCATIVA: ESCUELAS RURALES PARA UN DESARROLLO SOCIALMENTE SOSTENIBLE DEL CAMPO.
}

Tradition and continuity as an educational innovation: Rural schools for socially sustainable rural development.

Tradição e continuidade como inovação educacional: escolas rurais para o desenvolvimento socialmente sustentável do campo

Este trabajo forma parte de un proyecto titulado "Cambios sociales y mundos de vida rurales: hacia la sostenibilidad social y la cohesión territorial”, financiado por el MINECO (Ref. CSO2015-68696-P. 2016-2019).

\section{Óscar Fernández Álvarez}

Universidad de León, España. Teléfono: +34 987291416. Correo electrónico: oscar.fernandez@unileon.es

\section{Resumen}

El medio rural está en proceso de liquidación, tanto desde el punto de vista social como económico, y aunque diversos movimientos neo-rurales tratan de revertir esta tendencia, hasta el momento, estadísticamente es poco significativa. En este contexto, el objetivo de este trabajo es aportar evidencias del papel de las escuelas rurales como integrante del capital social para la continuidad y desarrollo socialmente sostenible del medio rural. La metodología utilizada es cualitativa, con un significativo trabajo de campo etnográfico realizado principalmente en Castilla y León. Asimismo, trataremos de comparar nuestros resultados con la literatura que documenta casos de éxito en otros países. Los resultados y discusión revelan los beneficios comunitarios de la escuela rural, además de que los resultados académicos, comparativamente, suelen ser mejores que en otras zonas. Las conclusiones muestran la necesidad de un compromiso institucional para convertir la escuela rural en un elemento imprescindible para la sostenibilidad social del campo y, en definitiva, para garantizar su continuidad futura.

Palabras clave: Escuela rural; sostenibilidad social; medio rural; innovación educativa

Tradición y continuidad como innovación educativa: Escuelas rurales para un desarrollo socialmente sostenible del campo 


\begin{abstract}
The rural environment is in the process of being liquidated, both socially and economically, and although several neo-rural movements try to reverse this trend, so far, statistically is not significant. In this context, the objective of this paper is to provide evidence of the role of rural schools as an integral part of social capital for the continuity and socially sustainable development of the rural environment. The methodology used is qualitative, with significant ethnographic field work done mainly in Castilla y León. Likewise, we will try to compare our results with the literature documenting cases of success in other countries. Results and discussion reveal the community benefits of the rural school, in addition to academic outcomes results are comparatively better than in other areas. The findings show the need for an institutional commitment to make the rural school an essential element for the social sustainability of the field and, in short, to guarantee its future continuity.
\end{abstract}

Keywords: Rural school; social sustainability; rural environment; educational innovation

\title{
Resumo
}

As zonas rurais está em liquidação, social e economicamente, e apesar de vários movimentos neo-rurais tentar inverter esta tendência, até agora, é estatisticamente insignificante. Neste contexto, o objetivo deste trabalho é fornecer evidências do papel das escolas rurais como membro do capital para a continuidade e desenvolvimento socialmente sustentável das zonas rurais. A metodologia utilizada é qualitativa, com significativo trabalho de campo etnográfico realizado principalmente em Castilla y León. Além disso, tentamos comparar nossos resultados com as histórias de sucesso literatura que documenta em outros países. Resultados e discussão revelam benefícios para a comunidade de escola rural, além de desempenho acadêmico, comparativamente, são geralmente melhor do que em outras áreas. Os resultados mostram a necessidade de um compromisso institucional para tornar a escola rural um elemento essencial para a sustentabilidade social do campo e, em suma, para garantir a continuidade do seu futuro. Palavras-chave: Escola rural; sustentabilidade social; ambiente rural; inovação educacional

Tradición y continuidad como innovación educativa: Escuelas rurales para un desarrollo socialmente sostenible del campo 


\section{Introducción}

Los programas de desarrollo rural, incluidos los de la UE, reflejan los esfuerzos para mejorar el bienestar económico, social y ambiental de los pueblos. Sin embargo, las instituciones y especialistas que los ejecutan se han centrado en la rentabilidad económica y se han olvidado de la naturaleza interdependiente de esas tres dimensiones. Las evidencias sugieren que para que los esfuerzos de desarrollo rural tengan éxito a largo plazo deben sumar además, el capital social (Putnam, 1993).

La escuela es fundamental en ese capital social, pero, en línea con lo señalado, se ha visto como mero recurso educativo $y$, consecuentemente, donde se cierra la escuela rural sucede la muerte del pueblo. Los docentes, para suplir las carencias que tiene el entorno, están en constante innovación. Este hecho, entre otros, podría explicar porqué Castilla y León, en el último informe PISA (2015) muestra buenos resultados. Recordemos la alta tasa de escuelas rurales de esta Comunidad, pues tiene el 50 \% del alumnado de infantil y primaria en colegios rurales.

\section{Objetivos y método}

Nuestro objetivo es analizar las escuelas rurales en una doble vertiente, como integrante del capital social para la continuidad y desarrollo sostenible en medio rural, y como entorno de aprendizaje innovador y de calidad gracias al papel de sus docentes. La metodología utilizada es cualitativa, con un significativo trabajo de campo etnográfico realizado principalmente en Castilla y León, con personal docente, padres y estudiantes de CRAs (Centro Rural Agrupado) de León, Soria y Ávila. Asimismo, tratamos de comparar nuestros resultados con la literatura que documenta casos de éxito en otros países

\section{Resultados y discusión}

La imagen nostálgica del "maestro de pueblo", que vivía dentro de la escuela hace tiempo que ha dejado de existir, pero no las dificultades de su práctica profesional. En cambio, las ventajas para los niños son mayores pues se benefician por ejemplo de una enseñanza muy individualizada, aprenden competencias en el lugar de trabajo, desarrollan relaciones significativas con los adultos y establecen relaciones de colaboración con sus respectivos pueblos. Ángel, (profesor CRA en Ávila) manifiesta: "tienen buenos referentes y muy cercanos, ya sea en sus propios compañeros, u otras Tradición y continuidad como innovación educativa: Escuelas rurales para un desarrollo socialmente 
personas, el panadero, el cartero o la propia naturaleza”. Se abre así el camino para que los estudiantes participen en experiencias de aprendizaje basadas en la comunidad donde los estudiantes, trabajando junto a los adultos, ganan una apreciación creciente de su entorno.

Variables como el tamaño o la ubicación de la escuela, máxime en situaciones de “ruralidad profunda” (Di Méo, 1998), afectan a la educación, al currículo o a los resultados. Al respecto, Alberto (profesor CRA en Soria), apunta: “en el mismo aula conviven niños de diferentes edades, los pequeños se benefician de los que aprenden los mayores, pero lo más importantes es que para eso, primero aprenden valores antes que contenidos”. Las investigaciones revelan que los estudiantes de las escuelas rurales enfrentan muchas dificultades educativas, desde vivir en territorio de exclusión hasta tener menos oportunidades o sofisticación tecnológica (Bouck, 2004).

Examinando nuestro trabajo en perspectiva transnacional, Dowling (2009) destaca en las escuelas rurales escocesas un enfoque comunitario y, más allá del curriculum nacional, y señala la importancia dada a la educación para la salud. Hargreaves, Kvalsund y Galton (2009) inciden en las diferencias que hay entre Inglaterra, donde las escuelas rurales son vistas como costosas e ineficientes, como un artículo de lujo más que una necesidad, y Noruega, donde los políticos trabajan para retener a las pequeñas escuelas rurales en una política de descentralización educativa. Aunque este segundo caso pudiera asimilarse a lo que sucede en nuestro territorio, no se lograría por la falta de confluencia de otros servicios e infraestructuras necesarias para la sostenibilidad de los pueblos.

Amiguinho (2015) apunta que las escuelas rurales en Portugal han trascendido los límites del sistema escolar convirtiéndose en signo de identidad y de existencia de las comunidades, sobreviviendo al intento de invisibilizarlas, algo constatable en nuestro territorio, pues todos los sectores lo tienen claro cuándo afirman: "mientras los niños se matriculen, el cole no cerrará”.

Por su parte, Hudson y Hudson (2008), señalan la importancia de la formación de los docentes para enfrentarse al trabajo en las escuelas rurales, dándoles de recursos y proporcionándoles experiencias de primera mano para revolver esa situación. En nuestro trabajo de campo hemos podido constatar que estos profesionales, no son 
profesores al uso porque un abismo separa la escuela rural de la urbana. Sus argumentos ilustran este hecho. Alberto de un CRA de Soria dice: "Esto te tiene que gustar porque es mucho trabajo. Tengo que preparar tres clases independientes para una hora. Pero los niños son muy agradecidos, y a diferencia de mi experiencia en una escuela de ciudad, aquí cualquier iniciativa a acogen de maravilla”.

\section{Conclusiones}

Los trabajos de Miller (1995) y sus continuadores han mostrado que las escuelas rurales, trabajando en asociación con locales y residentes, pueden tener un impacto positivo en la viabilidad de las comunidades rurales. Esto se muestra especialmente evidente en nuestro trabajo de campo, pues los estudiantes, trabajando junto a los adultos, tienen oportunidades significativas de participar en un aprendizaje comunitario que satisface las necesidades futuras de la comunidad, al tiempo que se ocupa de sus necesidades actuales de aprendizaje. La escuela, como capital social de la comunidad, no sólo ayuda a formar ciudadanos responsables, sino que también crea oportunidades para los adultos del mañana. Sin embargo, sin una fuerte colaboración entre organizaciones comunitarias, líderes locales y apoyo institucional que dote de bienes y servicios al medio rural es probable que las iniciativas de éxito no perduren en el tiempo. Por último, es imprescindible incidir en la formación de los docentes para cambiar la actitud para la enseñanza y la vida en las zonas rurales, en la vía de lograr un medio más socialmente sostenible.

\section{Referencias}

Amiguinho, A. (2015). La escuela, futuro del medio rural. Cuadernos de Pedagogía, 459

Boix, R. (2003). Escuela rural y territorio: entre la des ruralización y la cultura local. Revista Digital eRural, Educación, cultura y desarrollo rural, 1(1), 1-8.

Bouck, E. C. (2004). How Size and Setting Impact Education in Rural Schools. The Rural Educator, 25(3), 38-42.

Di Méo, G. (1998). Géographie sociales et territories, Paris: Nathan Université.

Dowling, J. (2009). Changes and challenges: Key issues for Scottish rural schools and communities. International Journal of Educational Research, 48, 129-139.

Tradición y continuidad como innovación educativa: Escuelas rurales para un desarrollo socialmente sostenible del campo 
Hargreaves, L., Kvalsund, R., \& Galton, M. (2009). Reviews of research on rural schools and their communities in British and Nordic countries: Analytical perspectives and cultural meaning. International Journal of Educational Research, 48, 80-88.

Hudson, P. \& Hudson, S. (2008). Sustainable futures for rural education: Changing preservice teachers' attitudes for teaching in rural schools. In: Australian Association of Research in Education (AARE) Conference 2008, 30 ${ }^{\text {th }}$ November 4th December, 2008, Brisbane. http://eprints.qut.edu.au/

Miller, B. (1995). The role of rural schools in community development: policy issues and implications. Portland, OR: Northwest Regional Lab., Rural Education Program.

PISA (2015) Pisa 2015 Castilla y León. Avance de resultados. Valladolid: Dirección General de Innovación y Equidad Educativa, Junta de Castilla y León.

Putnam, R.D. (1993). The prosperous community: Social capital and public life. The American Prospect, 13, 35-42.

Tradición y continuidad como innovación educativa: Escuelas rurales para un desarrollo socialmente sostenible del campo 